\title{
STRAIN-SOFTENING MATERIALS AND FINITE-ELEMENT SOLUTIONS
}

\author{
Ted Belytschko. Zdenek P. Bazant. Yll-Woong Hylin, and Ta-Pexg Chang \\ Department of Civil Engineering. Northwestern University. Evanston, IL 60201. L'S.A.
}

\begin{abstract}
Closed form and finite-element solutions are examined for several problems with strainsoftening materials. In the closed form solutions, strain-softening causes localization of the strain which is accompanied by an instantaneous vanishing of the stress. The finite-element solutions agree closely with analytic solutions in many cases and exhibit a rate of convergence only slightly below that for linear problems. The main difficulty which has been identified in strain-sottening constitutive models for damage is the absence of energy dissipation in the strain-softening domain. and this can be corrected by a nonlocal formulation. Finite-element solutions for the converging spherical wave problem exhibit multiple points of localization which change dramatically with mesh refinement. With a nonlocal material formulation, this pathology is eliminated.
\end{abstract}

\section{INTRODLCTION}

In materials such as concrete or rock. failure occurs by progressive damage which is manifested by phenomena such as microcracking and void formation. In most engineering structures, the scale of these phenomena. as compared to the scale of practical finite-element meshes, is usually too small to be modeled and their effect must be incorporated in the numerical analysis through a homogenized model which exhibits strain softening.

Strain softening. unfortunately, when incorporated in a computational model, exhibits undesirable characteristics. In static problems. finite-element solutions with strain softening often exhibit a severe dependence on element mesh size because of the inability of the mesh to adequately reproduce the localization of strain which characterizes static strain softening[1]. Furthermore. the solutions are physically inappropriate in that with increasing mesh refinement the energy dissipated in the strainsoftening domain tends to zero[2].

It was first hoped, although in retrospect little practical evidence existed for this optimism, that in dynamic problems. strain softening would not be as troublesome because the inertia of the continuum would alleviate the instability. Support for this can be found in the snapthrough of an arch: in this problem the load-deflection curve contains a limit point after which the force-deflection curve is negative. or softens. A static solution for the snapthrough is often very difficult. whereas a dynamic solution is relatively straightforward because the inertia of the structure alleviates some of the difficulties introduced by the negative slope in the force-deflection relation. The use of strain-softening models has become quite commonplace in dynamic concrete analysis. For example, in Marchertas et al.[3], strain softening appeared to reproduce the salient leatures of dynamic response of a concrete reactor vessel even after severe failure had developed. In the com- munity as a whole, a certain complacency evolved and except for the work of Bażant and colleagues[1, 2]. little effort was devoted to examining the basic soundness of numerical solutions with strain-softening materials.

Attention was recently focused on the validity of strain-softening models by the work of Sandler and Wright[4]: they asserted that strain-softening models are basically ill posed because a small difference in load results in large changes in the response. Sandler's example. which will be described in more detail later, consists of a one-dimensional rod with the velocity prescribed at one end in which the material strain softens. By increasing the load slightly, a significantly different response was obtained for the problem. Sandler and Wright also noted a strong dependence of the solution on the mesh size. They concluded that "a rate-independent dynamic continuum representation of strain softening is incapablc of reproducing softening behavior in a dynamic simulation of experiments" and then proceeded to show that in this problem the introduction of viscosity eliminates the sensitivity of the response to the load. Incidentally. as will be shown later in this paper, viscous damping is not a panacea for the sensitivity observed in strain-softening solutions: in certain problems which will be described herein. sensitivity to mesh size persists even after the introduction of damping.

In an effort to develop a problem with strain softening in which the localization does not occur on the boundary, we investigated two problems: one, which was presented in Ref. [5]. consists of a linear elastic bar joined to a strain-softening bar. Solutions for this bar were obtained by the method of images and compared to finite-element solutions. These results exhibited convergence with decreasing element length. Bažant and Belytschko[6] constructed a more interesting problem in which tensile waves are initiated at two ends of a bar so that strain softening occurs at the center. It was shown that a so- 
lution to this problem exists but that the behavior of the strain-softening domain is rather unusual: the strain softening localizes to an infinitely thin domain. a sel of measure zero. in which the strain becomes instantaneously infinite and in which the energy dissipation is zero.

In order to remedy some of these undesirable features of strain-softening solutions. Bažant. Belytschko, and co-workers[7,8] proposed a new nonlocal formulation for treating strain softening. Nonlocal theories have been introduced by Kröner[9]. Krumhansl[10] and Kunin[11], and developed further by Eringen and co-workers[12-14] and Edelen[15]. The basic ingredient of a nonlocal theory is that the stress-strain relation is not considered to be local but relates the stress at any point to the state of deformation within a finite volume about that point. In this respect. the theory lends itself admirably to problems of heterogeneous media. where the representation of the microscopic detail of strain fields and cracks is an insurmountable task. By dealing with an average of the strain over a linite domain about each point, the heterogeneity can be neglected, and the dispersion which occurs in inhomogeneous materials can be modeled without any artitices. It should be noted that in the transition from a microstructural theory to a macrotheory. strains and stresses are often averaged over finite volumes. However, this averaging is only considered in deleloping the macroconstitutive equation. In the solution of the governing equations, the constitutive equations are considered to apply only to points so the theory is local and the localization limiting property of nonlocal theories. i.e. the attribute of the theory which prevents localization to a set of measure zero, is then lost and the energy dissipation associated with strain softening vanishes.

An obvious question which arises is why one would want to introduce this complication in order to deal with strain softening. The reason for this is that when the constitutive equations are applied locally at points, then, as will be described here. no dissipation of energy occurs in the strain-softening domain because it is a set of measure zero. Thus the material can fail without any permanent dissipation of energy, which is physically quite unrealistic. By introducing a nonlocal character into the constitutive law. it is possible to restrict the localization to a domain of finite size just as is observed experimentally. and to achieve a finite amount of energy dissipation in the strain-softening domain.

However. we found we could not simply extend the existing nonlocal models to account for strain softening $[7,16]$. The existing nonlocal laws are not even self-adjoint. so they did not lead to symmetric stiffiness matrices. This lack of symmetry was found to be undesirable and was corrected by introducing the same averaging operation over the stresses as that for the strains. Such clouble averaging is in fact required by a consistent application of the varia- tional principle[8]. It Was also found that the train softening could only be introduced in the nonlocal law in a subtle tashion, necessitating a split of the constitutive equation into a local and nonlocal law. with the strain softening included only in the nonlocal portion. Numerical experiments indicated that without this particular combination. numerical solutions were often unstable.

The nonlocal law as introduced in Rets. [7. 8] offers substantial promise in providing well-posed solutions for heterogeneous materials that are subjected to damage and hence strain softening. There are however. substantial breakthroughs that yet need to be achieved: (1) efficient implementations of nonlocal laws in the finite-element method: (2) design of experimental methods for identifying the local and nonlocal portions of constitutive latis: and (3) methods for reconciling the bitureation between local damage. i.e. microcraching. and largescale fracture of a cleavage type in heterogeneous materials. However, the work reported here has shed light on the questions of numerical modeling of structures in the failure regime when strain softening takes place and provides the basis for future work.

We have organized the material as follows: in Sec. ? we describe several of the generic one-dimensional problems which can be used to examine the mathematical character of dynamic strain-softening solutions. In Sec. 3, the nonlucal continuum law will be described. In Sec. 4. some tinite-element solutions are presented for planar problems that indicate that the solutions converge to the analytic solution; however. only for a nonlocal law can finite energy dissipation be achieved in a strain-softening domain. In Sec. 5, local and nonlocal solutions will be given for the converging spherical and cylindrical wave. followed by conclusions in Sec. 6 .

\section{AVALYTICAL SOLLTION FOR STRIIN-SOFTENIYG MATERLAIS}

The problem by Sandler and Wright[4] is shown in Fig. 1. The essence of their argument was that the solutions are very sensitive to the constant i'b, which represents the maximum prescribed velocity at the left-hand boundary. and that the solution changes markedly and so does not appear to converge as the mesh is refined. Although the SandlerWright stress-strain law is nice from the viewpoint

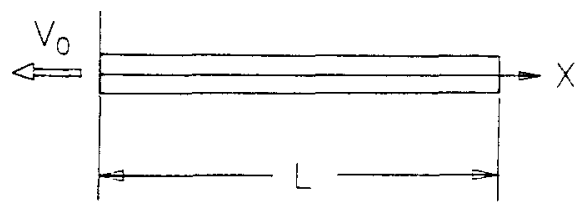

Fig. I. Sandter-Wrightl4] problem: the itress-stlain lat is $\left.\sigma=E_{0} \in \exp -\epsilon / \epsilon_{0}\right) . \epsilon_{0}=0.002 .11 .10 .11=1{ }^{2}{ }_{10} \mid 1-$ $\cos (\pi / t(1))$ for $t<t_{1}, t_{1}(0 . t)=i_{1}$ tor $t<t_{1} . t_{1}=0.2$ ms. 
that it provides a continuous relationship between stress and strain in the loading domain, it is not amenable to any attempts at a closed form solution by $d$. Alembert methods because of the dispersive character of the wave solution even in the loading range. For this reason, we have limited our studies to piecewise linear prescribed velocities or stresses and stress-strain law's of the type shown in Fig. 2: this will be called the moditied Sandler-Wright problem. Note that the stress goes to zero as the strain becomes large on the strain-softening sides of the law (usually the tensile side).

The analytical solutions to this problem are developed next. The salient characteristic of the analytical solution is the appearance of an infinite strain on the boundary once the strain $\epsilon_{p}$, is exceeded. The construction of the solution for this case will follow the approach presented by Bažant and $\mathrm{Be}$ lytschko[6] for a similar problem. As will be seen. when strain softening occurs. then the strain immediately localizes and reaches infinity within a time interval that approaches zero. Therefore, the solution can be generated by adding an image wave which cancels the incident wave so that the strainsoftening point is instantaneously converted to a free boundary. lows:

The governing equations can be stated as fol-

$$
\begin{aligned}
& \sigma_{\cdot .}=\rho u_{.,} . \\
& \sigma_{, \ell}=E(\epsilon) u_{,, r}=E(\varepsilon) \epsilon_{, \ell} .
\end{aligned}
$$

where $\sigma$ and $\epsilon$ are the stress and strain, "the displacement, and subscripts preceded by commas denote differentiation: $\rho$ is the density and $E$ the tangent modulus. We will consider two types of boundary conditions on the left-hand side, $x=0$ :

$$
\begin{array}{ll}
\text { velocity condition: } & \|,,(0, t)=-i^{\prime}{ }_{0}\langle t\rangle . \\
\text { traction condition: } & \sigma(0, t)=\sigma_{0}\langle t\rangle .
\end{array}
$$

where $\langle f\rangle$ designates $f H(f) . H$ being the Heaviside step function. The velocity boundary condition will be considered first. The right-hand boundary is as-

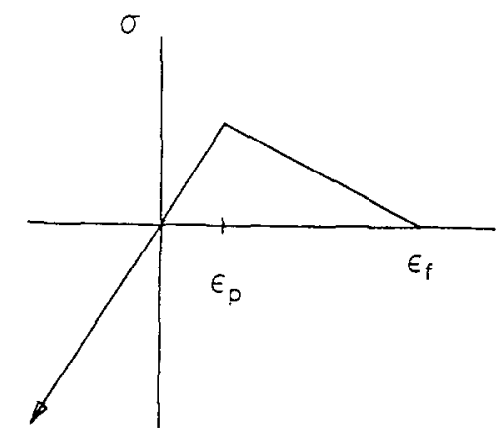

Fig. 2. Stress-strain law with strain softening showing nomenclature. sumed to be sufficiently far so that the rod can be considered semi-intinite.

Note that prior to the onset of strain softening. the problem is governed by the standard one-dimensional wave equation

$$
u \cdot \ldots=\frac{1}{c^{2}} u_{n} \cdot
$$

where

$$
\therefore=\frac{E}{\rho} \text {. }
$$

Once the strain-softening regime of the material is attained, then at those points the governing equation is

$$
\begin{gathered}
\bar{c}^{2} u_{1} \ldots+u_{\cdot u}=0 . \\
\bar{c}^{2}=-\frac{E}{\rho} .
\end{gathered}
$$

and $\bar{c}$ vanishes once $\epsilon_{p}$, is attained. Equation (2.7) is elliptic in space-time, which is peculiar in that information can be transmitted at infinite speeds. Hadamard[17] commented on this in 1903 and he claimed that the negative character of the square of the wave speed precluded its applicability to real materials since the wave speed would then be imaginary. However, the case of $c=0$ has been treated extensively by Taylor[18]. who noted that for perfectly plastic solids the deformation is localized at the point of impact. Wu and Freund[19] have recently presented a lucid description of these localization phenomena and investigated the effects of strain-rate sensitivity and heat transfer on the localization. However, the analyses were limited to the case where in the limit $c=\bar{c}=0$.

We will here consider the strain-softening situation using the concepts developed in [6]. The present situation differs from [6] in that the stress wave is a ramp rather than a step wave, but it will be found that all of the singularities associated with a step input remain.

The procedure of constructing a solution consists of three steps:

(1) It is shown that the boundary between the strain-softening and elastic domain cannot move. so the strain-softening domain is limited to a point.

(2) This is shown to imply that the strain and strain rate in the strain-softening points must be infinite.

(3) Since the strain rate is infinite for the class of materials considered here. in which $\sigma \rightarrow 0$ as $\epsilon \rightarrow x$. the stress can be considered to vanish instantaneously at the points which strain soften.

The last conclusion enables the solution to be easily constructed by the d'Alembert method by 
simply adding a wave to satisty the zero stress condition.

For the prescribed velocity problem. let $t$, be the time when the left-hand end. $x=0$. reaches $\epsilon_{r}$, and begins to strain soften: $t$ is given by

$$
t_{1}=\frac{2\left(\hat{E}_{i}\right)}{\left.i_{i}^{\prime}\right)}
$$

and the solution prior to the onser of strain softening is given by

$$
\begin{aligned}
& u=-\frac{i^{2} u}{3}\left\langle\left(1-\frac{x}{c}\right)^{2}\right\rangle . \\
& \epsilon=\frac{i^{\prime} t}{c}\left\langle t-\frac{x}{c}\right\rangle .
\end{aligned}
$$

Strain softening first occurs at $x=0$. We now show that the boundary between the elastic and the softening interface cannot move. For this purpose. the usual formula for velocity $V$ of discontinuities is used (a development is given in [6]):

$$
\sigma^{+}-\sigma^{-}=\rho V^{2}\left(\epsilon^{+}-\epsilon^{-}\right) \text {. }
$$

where the superscripts + and - designate the state variables to the right and left of the discontinuity. respectively. If the material is strain softening behind the intertace and not yet before it, it follows that $\epsilon^{-}>\epsilon^{-}$and $\sigma^{+} \geqslant \sigma^{-}$. Substituting these inequalities into eqn (2.12), it follows that $v^{2}$ must be negative or zero: since the former assumption would yield an imaginary velocity for the discontinuity, only $V=0$ is tenable, and it can already be concluded that

$$
\sigma^{+}=\sigma^{-}
$$

To slow that the strain and strain rates must be infinite at a point which strain softens, a solution is constructed in the strain-softening domain which is considered to be $0 \leqslant x \leqslant s$ where $s \rightarrow 0$. It can be seen that

$$
\begin{aligned}
u & =u^{*}+\left[a\left(i-t_{1}\right)+\epsilon_{p}\right] x . \\
u^{*} & =-\frac{i^{\prime} u}{2}\left(\left(u_{1}-\frac{x}{i}\right)^{2}\right)
\end{aligned}
$$

satisfies the governing equation in the strain-softening domain. (2.7). This solution. 12.14), is now matched to a solution in the elastic domain

$$
\begin{aligned}
& u=-\frac{i n}{2}\left(\left(t-\frac{x}{c}\right)^{2}\right)+f(\xi) H\left(t-t_{1}\right) . \\
& \xi=t-\frac{x}{c}-t_{1} .
\end{aligned}
$$

where the second term is a wave emanating in the strain-softening region which will be used to match the displacements and stress conditions across the interface. From eqn (2.14a), it follows that

$$
\epsilon^{-}=\frac{\partial u}{\partial x}=\left(a\left(t-t_{1}\right)+\epsilon_{m} .\right.
$$

If the two displacement solutions, eqns (2.14a) and (2.15), are now matched across the interface $x=s$, then

$$
\begin{gathered}
u^{*}+\left[a\left(t-t_{1}\right)+\epsilon_{r}\right] \cdot s \\
=-\frac{\left.i^{\prime \prime}\right)}{2}\left\langle\left(t-\frac{s}{c}\right)^{2}\right\rangle+f(\bar{\xi}) . \\
\bar{\xi}=t-\frac{s}{c}-t_{1} .
\end{gathered}
$$

Eliminating $a$ from eqns (2.17) and (2.18) yields

$$
\epsilon^{-}=\frac{1}{s}\left[f(\bar{\xi})-\frac{i v}{2}\left(t-\frac{s}{c}\right)-u^{*}\right] .
$$

It can be seen that as $s \rightarrow 0, \epsilon^{-} \rightarrow 0$ instantaneously. which through eqn (2.13) implies $\sigma^{+}=0$. The function $f(\xi)$ is then found from this condition. Using the displacement field of eqn (2.15) and letting $\sigma^{+}$. and hence $\epsilon^{+}$, vanish, we find

$$
\begin{aligned}
f^{\prime}(\xi)=\epsilon_{p} c H\left(t-t_{1}-\frac{x}{c}\right) & \\
& +v_{0}\left(t-t_{1}-\frac{x}{c}\right\rangle . \\
f(\xi)=\epsilon_{p} c & \left\langle t-t_{1}-\frac{x}{c}\right\rangle \\
& +\frac{t^{\prime} t}{2}\left\langle\left(t-t_{1}-\frac{x}{2}\right)^{2}\right\rangle .
\end{aligned}
$$

Hence the complete solution is

$$
\begin{aligned}
& u=-\frac{v_{0}}{2}\left\langle t-\frac{x}{c}\right\rangle+\epsilon_{p} c\left\langle t-t_{1}-\frac{x}{c}\right\rangle \\
&+\frac{i_{0}}{2}\left\langle\left(t-t_{1}-\frac{x}{c}\right)^{2}\right\rangle \\
& u_{, t}=-\frac{v_{0}}{2} H\left(t-\frac{x}{c}\right)+\epsilon_{p} c H\left(t-t_{1}-\frac{x}{c}\right) \\
&\left.+i_{t}\left\langle t-t_{1}-\frac{x}{c}\right\rangle\right)
\end{aligned}
$$

This solution will subsequently be compared to finite-element solutions.

The solution for the stress boundary condition. eqn (2.4). can be found by replacing $z_{0}$ by $\sigma_{0} \cdot E$. However, in the prescribed stress form of this problem, eqn (2.4), the introduction of the image at the strain-softening point poses a difficulty since the first point to strain soften is initially on the boundary. Thus, in one sense it can be said that this 
boundary must satisfy two different boundary conditions: eqn (2.4) and $\sigma=0$.

This contradiction can only be reconciled by requiring the second boundary condition (that the stress vanishes) to take precedence. In fact the first boundary condition would be impossible to enforce in an experiment. The notion of a boundary condition depending on the result of the solution is not totally unexpected in an analysis of a continuum which fails. For example, in a buckling problem with unstable postbuckling behavior, the prescribed force would also be limited by the capacity of the structure. Yet the situation in the buckling problem is not completely analogous: in a dynamic buckling problem, any force may be prescribed and the excess force will generate accelerations. which depend on the magnitude of the force, whereas in this problem, the solution is completely independent of the value of the prescribed stress once the failure stress is exceeded. Nevertheless, this model does appear to represent a physically meaningful situation: the behavior of a rod in which the material can sustain a limited tensile strain before it fails. and the solution appears reasonable.

From a mathematical viewpoint, the character of the solution presents some other dilemmas. First of all, we consider $0<x<s$ to be a segment in developments of eqn (2.19), but it is only a point. Second. since the strain softening portion localizes to a point at which the displacement is discontinuous, within conventional theories for partial differential equations, the body would no longer be considered to be a single body: strain softening subdivides the initial body into two by introducing interior boundaries. Similarly in three-dimensional problems, the strain-softening domain is a surface. In both cases, these are sets of measure zero. Although mathematical theories for such partial differential equations are not known to us, there is no reason to arbitrarily exclude such phenomenological models.

Another difficulty posed by this model is that the energy dissipated in the formulation of the strain-softening region is not finite but instead vanishes. This can be seen from the fact that the only irreversible energy loss in the material shown in Fig. 2 occurs in the strain-softening domain. Because the strain-softening domain in a one-dimensional problem becomes a point, and since the energy dissipated per unit length is finite, the total energy dissipated vanishes. This in fact is a more serious difficulty than the mathematical difficulties, for the strain-softening constitutive equation is often intended to represent microcracking, which is a dissipative process. It will be seen that, in spite of the mathematical questions, the behavior of finite-element solutions is not pathological.

Remarks: (1) The solution is puzzling when the ramp loads, eqns (2.3) and (2.4), are replaced by step functions. According to the present analysis, if $\sigma_{i}$ $>\epsilon_{p} E$, then the boundary point should reach strain softening instantaneously and no wave should reach the interior.

(2) The solution does not depend on the specific functional dependence of stress on strain in the strain-softening portion if the stress vanishes as the strain becomes large.

One conceptual difficulty of the Sandler-Wright problem is that strain softening occurs only at the boundary. which confuses the role of the boundary condition and the strain softening. For this reason we have attempted to construct problems in which strain softening occurs within the domain of the problem.

The strictly one-dimensional problems of this type are shown in Figs. 3 and 4. The first consists of an elastic rod joined to a rod with a strain-softening material[5]. We will not give the closed form solution but only explain its major features. If the applied stress is sufficiently large. then strain softening is initiated at the interface between the two materials. The strain localizes at this point. and as in the previous problem, the stress vanishes instantaneously at the interface. The solution can thus be viewed as a case in which a body separates into two.

The second problem, given in [6], consists entirely of a strain-softening material. Equal and opposite velocities in (or forces) are applied to the two ends of the bar, so that tensile waves are generated at the two ends. These propagate to the center: when they meet at the center, the stress doubles for elastic behavior, so strain softening is possible at this midpoint even though it did not occur at the boundaries.

The solution is given in [6] for prescribed velocities that are step functions in time. As in the previous case, localization occurs at the midpoint where the strain becomes infinite. The solution is symmetric about the midpoint $x=0$; for the left half $(x<0$ and $0 \leqslant t \leqslant 2 L / c)$

$$
\begin{gathered}
\left.u=v_{0}^{\prime}\left\langle t-\frac{x+L}{c}\right\rangle-v^{\prime}\right)\left\langle t-\frac{L-x}{c}\right\rangle \\
\epsilon=\frac{v_{0}}{c}\left[H\left(t-\frac{x+L}{c}\right)-H\left(t-\frac{L-x}{c}\right)\right. \\
+4(c t-L\rangle \delta(x)]
\end{gathered}
$$

where $\delta(x)$ is the Dirac delta function.

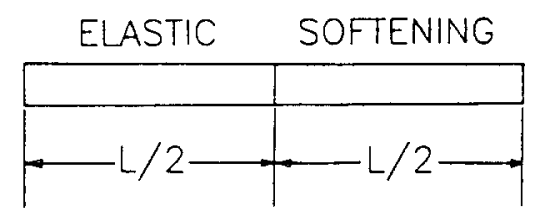

Fig. 3. Problem with strain softening at interface between elastic and softening domain (Ref. [5]). 


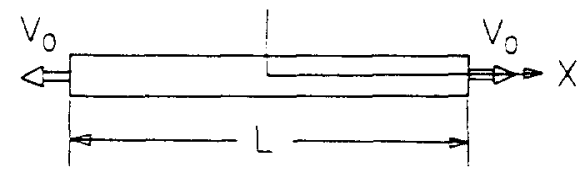

Fig. 4. One-dimensional problem in which strain softening vecurs at $x=0$ (Ref. $[6]$ )

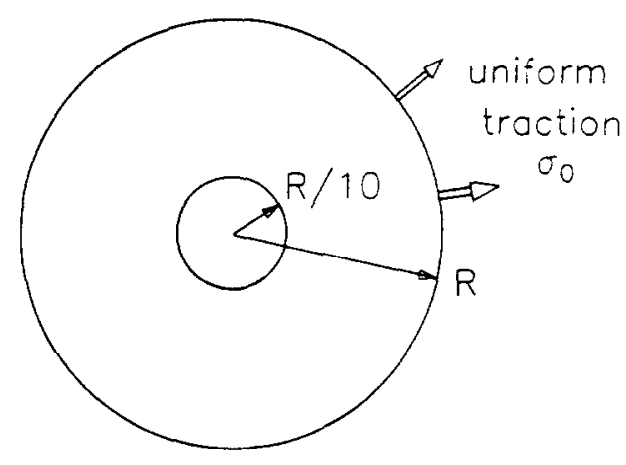

Fig. 5. Spherical converging wave problem.

Another problem we have considered is a sphere loaded on its exterior surface (see Fig. 5). This problem is not easily physically realizable with a tensile load: however, it is physically meaningful with a compressive load, and strain softening can occur in some materials in compression (although the stress usually will not vanish as the dilatation becomes large).

This problem has an intriguing feature. Consider a load which is a ramp function in time. Prior to the onset of strain softening at an interior surface, part of the wave can have passed through this surface. Since the stresses in the wave which are inside the initial surface of strain softening are amplified as the wave passes to the center. the formation of additional strain-softening surfaces is possible. As a result, this problem has considerably more structure than the one-dimensional problems.

\section{NONLOCAL, CONTINUUM FOR STRAIN SOFTENING;}

The major shortcoming of strain-softening models for representing local damage is that the localization phenomenon associated with strain softening results in no dissipation of energy. For example, in one-dimensional problems. the strainsoftening process is limited to a single point and since the rate of work per unit length is finite, the amount of work dissipated in the strain-softening domain vanishes. Analogously, in three-dimensional problems. strain softening localizes to a surface, and since the work per unit volume is again finite, the total energy dissipation due to the separation across the surface is finite. Thus. no energy is required to separate the material along the surface of strain softening. which is physically quite unrealistic. It should be stressed that all of these comments apply only to materials in which the stress across the surface tends to zero as the strain becomes large: if the stress tends to some nonzero value. then the response of the material may bo quite different and the dissipation no longer vanishes. However. any dissipation in excess of that which corresponds to the perfectly plastic dissipation associated with the final stress vanishes.

To achieve tinite energy dissipation during fail. ure by strain sottening. the artitice of imposing a ccrtain minimum element size which depends on the aggregate size (crack band model) has been proposed[2]. However, this artifice may be inconvenient in practical analysis. since it requires the element size to be dictated by a material constant rather than by the size of the structure.

In order to avoid this difficulty. we have investigated the possibility of using nonlocal constitutive laws in which the stress at a point is related to the weighted average of the strain in a neighborhood of that point. This is a special case of the existing (or classical) nonlocal continum theories $[9-14]$. However, it was found to be necessary to make two modifications of this theory in order to obtain realistic results in the strain-softening regime: (1) the existing nonlocal theory is not sell-adjoint and hence possesses certain spurious zero-energy modes of deformation: (2) in order to achieve stable solutions even in one dimension in the presence of strain softening with a constant weight, a material law consisting of a combination of a local law without strain softening and a nonlocal law with strain softening was required.

We will now sketch the essential features of this nonlocal theory for one-dimensional problems. Details may be found in Refs. 17.81. The fundamental assumption in a nonlocal theory is that the nonlocal strain $\bar{\epsilon}$ at a point is a weighted average of the strains in a neighborhood of that point. Thus

$$
\begin{aligned}
\bar{\epsilon}(x) & =\int_{x-12}^{1+12} \epsilon(x+s) n(s) \mathrm{d} s \\
& =\int_{x-12}^{1+12} \frac{\partial u}{\partial x}(x+s) u(s) d s .
\end{aligned}
$$

where $w(s)$ is a given weighting function. The stress-strain law is then written in terms of $\bar{\epsilon}$. and its rate form is

$$
\sigma_{. l}(x)=E(\bar{\epsilon}) \bar{\epsilon}_{. l}(x)
$$

Although the classical nonlocal theory directly uses the stress $\sigma$ in the momentum equation. eqn (2.1), the resulting form is not self-adjoint[16]. This leads to the existence of spurious, zero-energy modes of deformation for certain weighting functions $1(x)$ : deformations which are associated with vanishing strains $\bar{\epsilon}$ and hence do not generate any stresses. These spurious modes have been found for uniform (constant) weighting functions $u(x)$.

To remedy this difficulty. the stress o is pro- 
cessed through an operator identical to (3.1).

$$
\bar{\sigma}(x)=\int_{1-12}^{1-12} \sigma(x+s) w(s) \mathrm{d} s .
$$

and the resulting stress is used in the equation of motion. eqn (2.1). Once eqn (3.3) is added to the system. spurious modes are eliminated even for constant weighting functions $w(x)$. It was also shown by Bazant[8] that the averaging of $\sigma(x)$ is required within a consistent application of a variational principle such as the principle of virtual work. A nonlocal continuum which is characterized bv double averaging. once on the strains. as in (3.1) and then on the stresses, as in (3.3). is the limiting case of a series of imbricated (overlapping) finite elements. Therefore, this type of nonlocal continuum was termed an imbricated continuum by $\mathrm{Ba}$ Żant[8].

Even with a self-adjoint form of the nonlocal laws. solutions for strain-softening materials are unstable for constant weights $w(x)$. So far, only by combining a local and nonlocal law has stability been achieved[16, 7]. By superimposing two distinct field systems, one local and without strain softening, and a nonlocal law with strain softening, stability is achieved in a model which exhibits a negative slope for a finite segment.

The governing equations for this model can be summarized as follows:

$$
\begin{array}{ll}
\sigma_{. t}=E \bar{\epsilon}_{. t} & E \text { can be negative, } \\
\tau_{. t}=E \epsilon_{, t}, & E>0, \\
\text { eqn }(3.3): \sigma \rightarrow \bar{\sigma}, & \\
S=(1-\gamma) \bar{\sigma}+\gamma \tau, & 0<\gamma<1 . \\
S_{-\tau}=\rho l_{. t t} . &
\end{array}
$$

Finite element solutions for this nonlocal law are given in Sec. 5 for cylindrical and spherical geometries. These results were obtained with an imbricate finite-element model, and with various finite-element meshes. It will be seen that the strain distributions computed by the nonlocal law are quite well-behaved for various meshes, whereas the local formulation predicts strain that vary erratically with mesh refinement.

\section{FINITE-ELEMENT SOLUTIONS FOR PLANAR PROBLEMS}

Finite-element solutions for the moditied Sandler-Wright problem. Fig. I, obtained with the local material law given in Fig. 2. are shown in Fig. 6. Solutions were obtained with meshes of 50.100. and 200 elements. Linear displacement. constant strain elements, and lumped mass matrices were used. Time integration was performed with the cen. tral difference method.

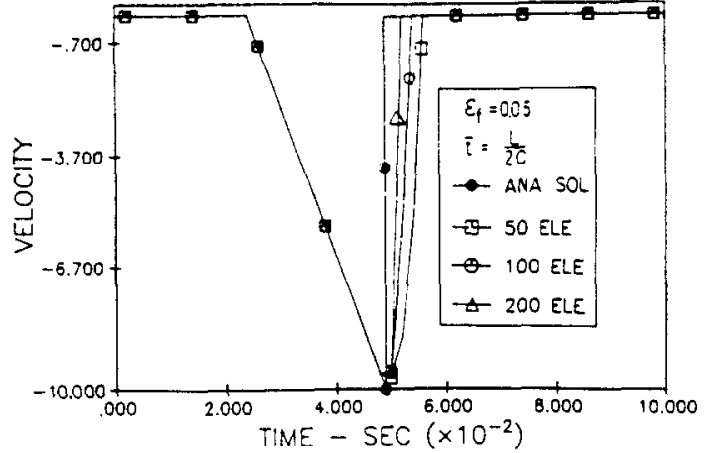

Fig. 6. Velocity-time history at $x=L / 4$ for geometry shown in Fig. 1, stress-strain law given in Fig. 2: $\epsilon_{p}=$ $0.01 . \epsilon_{t}=0.05 . L=100 . c=10^{3}$.

The finite-element solutions are compared with the analytic solution given in the Sec. 2. It can be seen that the agreement is quite good and improves with mesh refinement. although the instantaneous drop in the velocity, which is a result of the strain localization. cannot be reproduced even with the finest mesh.

The rate of convergence for the case when $\epsilon_{v}=$ $\epsilon_{r}=0.01$ is shown in Fig. 7. Here the error $a$ is defined by

$$
e^{2}=\int_{11}^{11} \int_{11}^{t}\left(t^{,}, 1 \mathrm{MN}-t^{, \mathrm{ANA}}\right)^{2} \mathrm{~d} x \mathrm{~d} t
$$

where $r$ with superscripts FEM and ANA are the finite-element and analytic velocities. respectively. As can be seen from Fig. 7. the rate of convergence is approximately proportional to $h^{1 . \text { th }}$ where $h$ is the element length. This is not much less than the the-

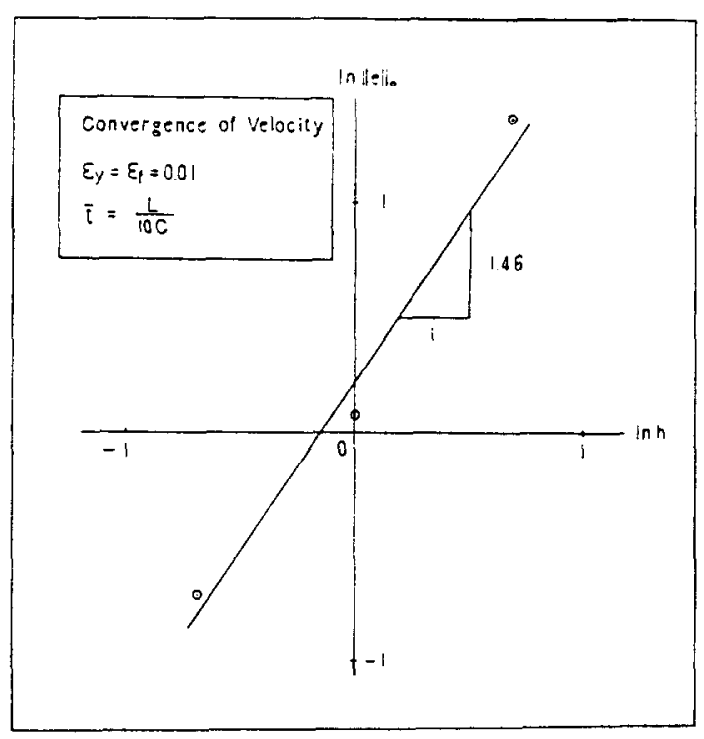

Fig. 7. Rate of convergence of finite-element solution for velocity to analytic solution for modified Sandler-Wright problem with $\epsilon_{1}=\epsilon_{1}=0.01$. 


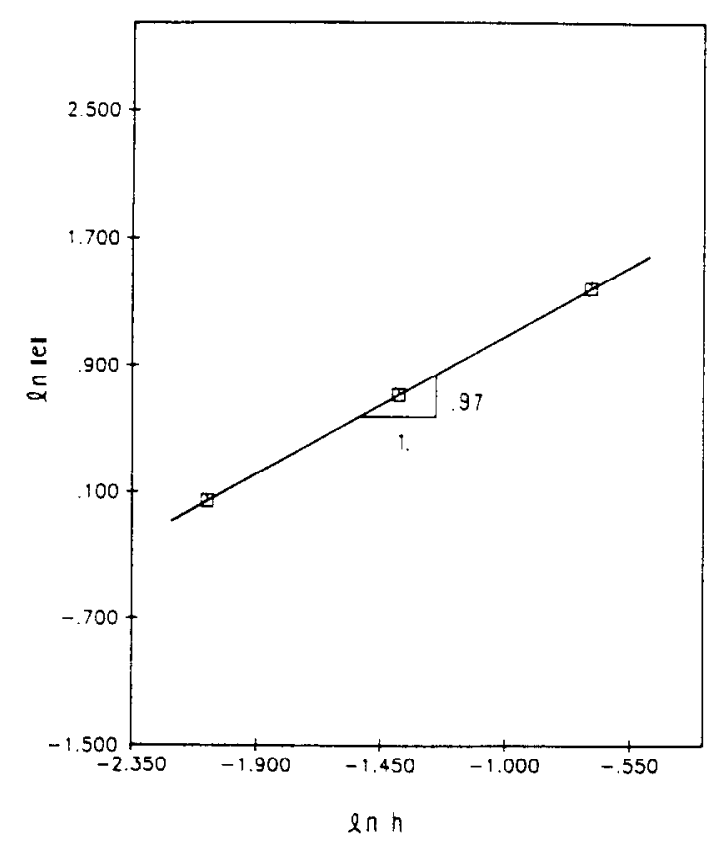

Fig. 8. Rate of convergence of finite-element solution for velocity to analytic solution for modified Sandler-Wright problem with $\epsilon_{y}=2 \epsilon_{i}=0.02$.

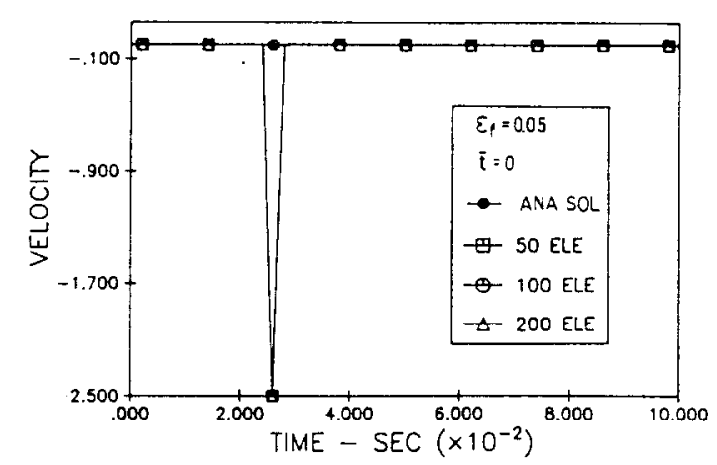

Fig. 9. Finite-element solution to modified SandlerWright problem with $p=p_{0} H(t)$. $H$ being the Heaviside step function.

oretical value of $h^{2}$ expected for linear solutions by these methods, so the sensitivity to meshing which Sandler and Wright pointed out is not evident. The rate of convergence for a material with $\epsilon_{f}=2 \epsilon_{y}=$ 0.02 is shown in Fig. 8; as $\epsilon_{i}$ increases, the rate of convergence deteriorates. Figure 9 shows the finiteelement solution when the input is a step function. Theoretically, there should be no waves inside the rod, but because of the finite size of the elements, a small pulse penetrates the mesh in a numerical solution.

\section{FINITE-ELEMENT SOLUTIONS FOR SPHERICAL AND CYLINDRICAL PROBLEMS}

As mentioned in Sec. 2, the converging spherical wave problem is particularly intriguing because it offers the possibility of strain softening being ini- tiated at many points by a wave. Although we have not been able to find a closed form solution for this problem. we have examined both local and nonlocal finite-element solutions. The local finite-element solutions exhibit radical differences as the mesh is refined, and this ostensible absence of convergence is not alleviated by the addition of damping. Nonlocal solutions are free of these difficulties. These solutions will be summarized in this section.

In spherical or cylindrical coordinates, the strain-displacement relations are

$$
\begin{array}{cc}
\epsilon_{x}=u, x, \epsilon_{y}=\frac{u}{x}, \quad\left\{\begin{array}{l}
\epsilon_{z}=\epsilon_{y} \text { (spherical), } \\
\epsilon_{z}=0 \text { (cylindrical), }
\end{array}\right. \\
\epsilon=\epsilon_{1}+2 \epsilon_{y} . \quad e_{x}=\frac{3}{3}\left(\epsilon_{x}-\epsilon_{y}\right), \\
e_{x}=\frac{1}{3}\left(\epsilon_{y}-\epsilon_{x}\right) & \text { (spherical). } \\
\epsilon=\epsilon_{x}+2 \epsilon_{y}, & e_{x}=\frac{1}{3}\left(2 \epsilon_{x}-\epsilon_{y}\right), \\
e_{v}=\frac{1}{b}\left(\epsilon_{y}-\epsilon_{x}\right) & \text { (cylindrical). }
\end{array}
$$

We denote by $\bar{e}_{x}, \bar{\epsilon}_{y}, \bar{\epsilon}, \bar{e}_{x}, \bar{e}_{y}$ the means of $\epsilon_{x}$, $\epsilon_{y}, \epsilon, e_{x}, e_{y}$, respectively; defined as in eqn (3.1).

The stress-strain law is

$$
\begin{aligned}
\sigma_{x, t} & =\overline{K \epsilon}_{, t}+2 \overline{G e}_{x, t}, \\
\sigma_{y, t} & =\overline{K \epsilon}_{, t}+2 \overline{G e}_{y, t}, \\
\tau_{x, t} & =K \epsilon_{, t}+2 G e_{x, t}, \\
\tau_{y, t} & =K \epsilon_{, t}+2 G e_{y, t,},
\end{aligned}
$$

and the total stress $S$ is obtained by

$$
\begin{aligned}
\bar{\sigma}_{x}(x) & =\int_{x-1 / 2}^{x+1 / 2} \sigma_{x}(x+s) w(s) \mathrm{d} s, \\
\bar{\sigma}_{y}(x) & =\int_{x-1 / 2}^{x+1 / 2} \sigma_{y}(x+s) w(s) \mathrm{d} s, \\
S_{x} & -(1-\gamma) \bar{\sigma}_{x}+\gamma \tau_{x}, \\
S_{v} & =(1-\gamma) \bar{\sigma}_{y}+\gamma \tau_{y} .
\end{aligned}
$$

The equation of motion is

$$
\begin{aligned}
& S_{x, . r}+\frac{2}{x}\left(S_{x}-S_{y}\right)=\rho u_{, \prime \prime} \quad \text { (spherical), } \\
& S_{x, r}+\frac{1}{x}\left(S_{x}-S_{y}\right)=\rho u_{, \prime \prime} \quad \text { (cylindrical). }
\end{aligned}
$$

Here $u=$ radial displacement (Fig. 10c): $\epsilon_{x}, \epsilon_{y}=$ radial and circumferential normal strains (lacal); $\epsilon$ $=$ volumetric strain; $e_{x}, e_{v}=$ deviatoric strains (local): $\tau_{x}, \tau_{y}=$ local radial and circumferential normal stresses: $\sigma_{x}, \sigma_{r}=$ broad-range radial and circumferential normal stresses: $S_{v}, S_{v}=$ total radial and circumferential normal stresses: $K \cdot G \cdot \bar{K} \cdot \bar{G}=$ local and broad-range (nonlocal) bulk and shear moduli. In eqns (5.4) and (5.5) isotropy of the material is assumed. The shear moduli $G$ and $\bar{G}$ are assumed to be constant. while $K$ and $\bar{K}$ depend on 


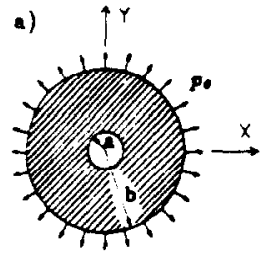

c)
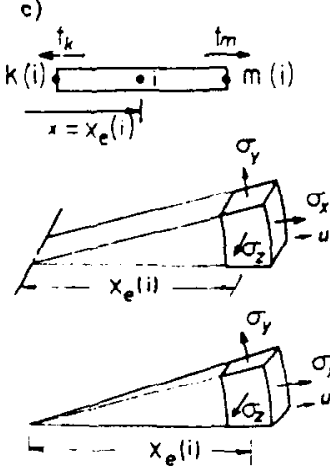

b)

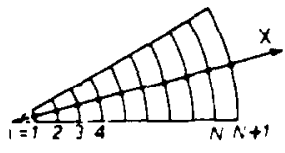

d)

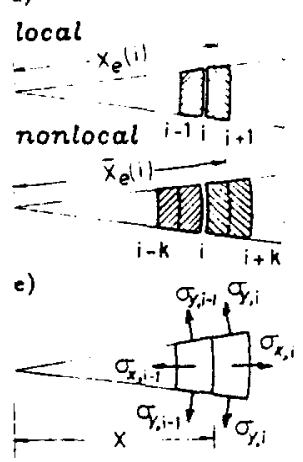

Fig. 10. Notation for spherical and cylindrical wave problems.

$\epsilon$ and $\bar{\epsilon}$, respectively: $K>0$ but $\bar{K}$ may become negative, which represents strain softening.

We consider waves generated by a sudden application of a uniform normal traction at the exterior surface of a hollow sphere or a hollow infinite cylinder. The traction is a Heaviside step function of time, so the boundary condition at $x=b$ (Fig. 10a) is $\sigma_{x}=p_{0} H(t)$, and the interior surface is loadfree, i.e. $\sigma_{x}=0$ at $x=a(a, b=$ internal and external surface radii). Initially, the body is at rest. The elastic solution[20], consists of a step wave with a strain which grows as the wave propagates toward the center.

The closed form solution is

$\|=\frac{b^{3} p_{0}}{4 G x^{2}}\left\{1-e^{\xi \tau}\left[\frac{\xi}{\omega}\left(\frac{2 x}{b}-1\right) \sin \omega \tau+\cos \omega \tau\right]\right.$

$$
\text { for } \tau \geqslant 0 \text {, }
$$

as may be checked by substitution in eqns (5.1)(5.8) with $\gamma=1, K=$ constant; $\tau=t-(b-r) /$ $c_{1}, \xi=2 c_{2}^{2} /\left(b C_{1}\right), \omega=\xi\left[\left(c_{1} / c_{2}\right)^{2}-1\right]^{1 / 2}, c_{1}=[3 K(1$ $-\nu) /(1+\nu) p]^{1 / 2}, c_{2}=(G / \rho)^{1 / 2}, v=(3 K-2 G)$ $(6 K+2 G)=$ Poisson's ratio.

For the numerical solutions, one-dimensional meshes with (Fig. 10b) two-node elements with linear displacements and a single quadrature point (at the element center) were used. The local elements are of length $h$ and the imbricate elements of length $l=n h$. The mass matrix is lumped.

The first group of numerical solutions was made with the aim of examining the character of the spherical wave solution and determining whether damping is sufficient to achieve well posedness, as in the planar one-dimensional problem[4].

Shear moduli $G$ and $\bar{G}$ are assumed to be neg. ligibly small $\left(10^{-6}\right)$. The following constants were used: bulk modulus $K=1.0$. density $\rho=1$. shear modulus $G=1 \times 10^{-6}, \epsilon_{1}=1.0 . \epsilon_{t}=5.0 \mathrm{isee}$ bottom of Fig. 11). Damping was added by a viscous stress defined by

$$
\sigma_{i j}^{v i v}=2 \eta(\rho K)^{1 / 2} \dot{\epsilon} \delta_{i j} .
$$

with $\eta=0.1$. This provided enough viscosity to damp the cutoff frequency of the mesh by $44 \%$ and $6 \%$ of critical damping in the fine and coarse meshes, respectively. In some solutions. the damping was turned off whenever $\epsilon_{f}$. the strain at which the stress vanishes. was reached. This is called a damping cutoff.

Comparing the results in Fig. 12 for the various meshes, one can see a striking difference in the results within the strain-softening domain. which is the domain to the left of what is marked as the transition point. As the mesh is refined, several points of localization develop and the points of localization change arbitrarily with mesh refinement even with damping. However, the displacement outside the transition surface appears to converge with mesh refinement.

Figure 13 shows a similar solution except that the damping was not cut off when localization developed. Again, the solution does not exhibit any pattern of convergence in the strain-softening domain. The stresses in the region near the transition point are quite different from those in Fig. 12, which employed a damping cutoff.

The next group of solutions examines the effect of the nonlocal formulation. In these solutions, the bulk behavior follows the bilinear total stress-strain diagram in Fig. 1la. characterized by elastic bulk moduli $\bar{K}=\bar{K}_{0}=1$, strain $\epsilon_{p}=1$ at peak stress and $\epsilon_{f}=5$ at the end of strain softening. This diagram is obtained by assuming that the local behavior is elastic-ideal plastic (Fig. IIb), and that the broad-range behavior (Fig. Ila) exhibits oversoft-

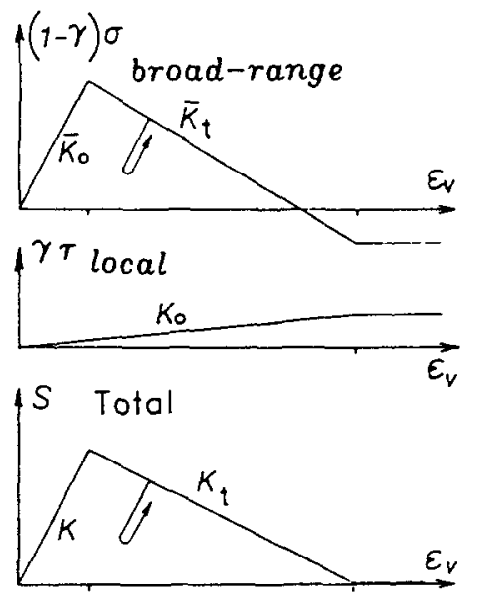

Fig. 11. Stress-strain law for spherical and cylindrical wave problems; only the lowest figure applies to local laws. 

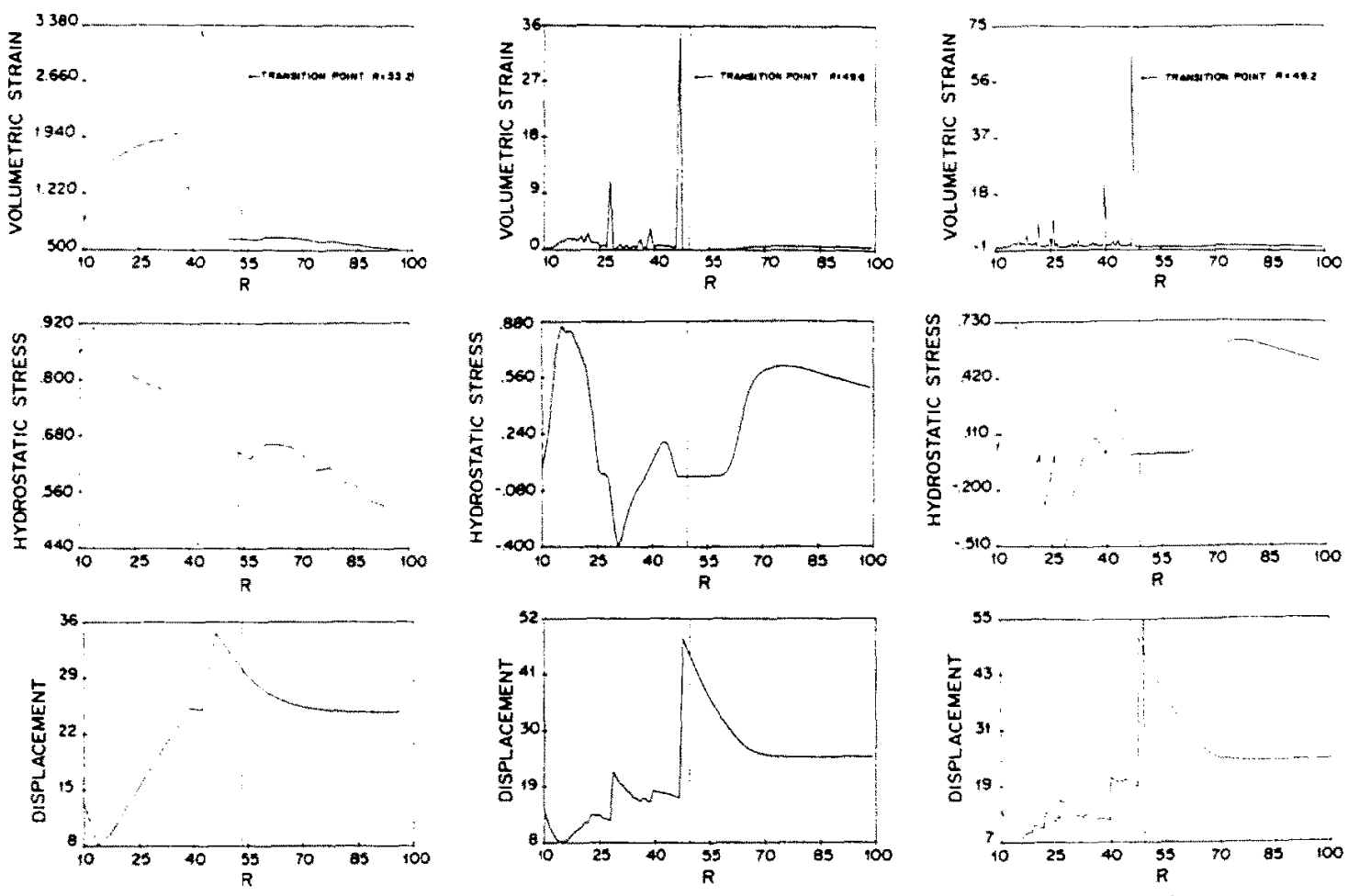

$(c)$

Fig. 12. Solutions to spherical wave problem for various meshes with damping for meshes of (a) 25 elements, (b) 100 elements, and (c) 200 elements.
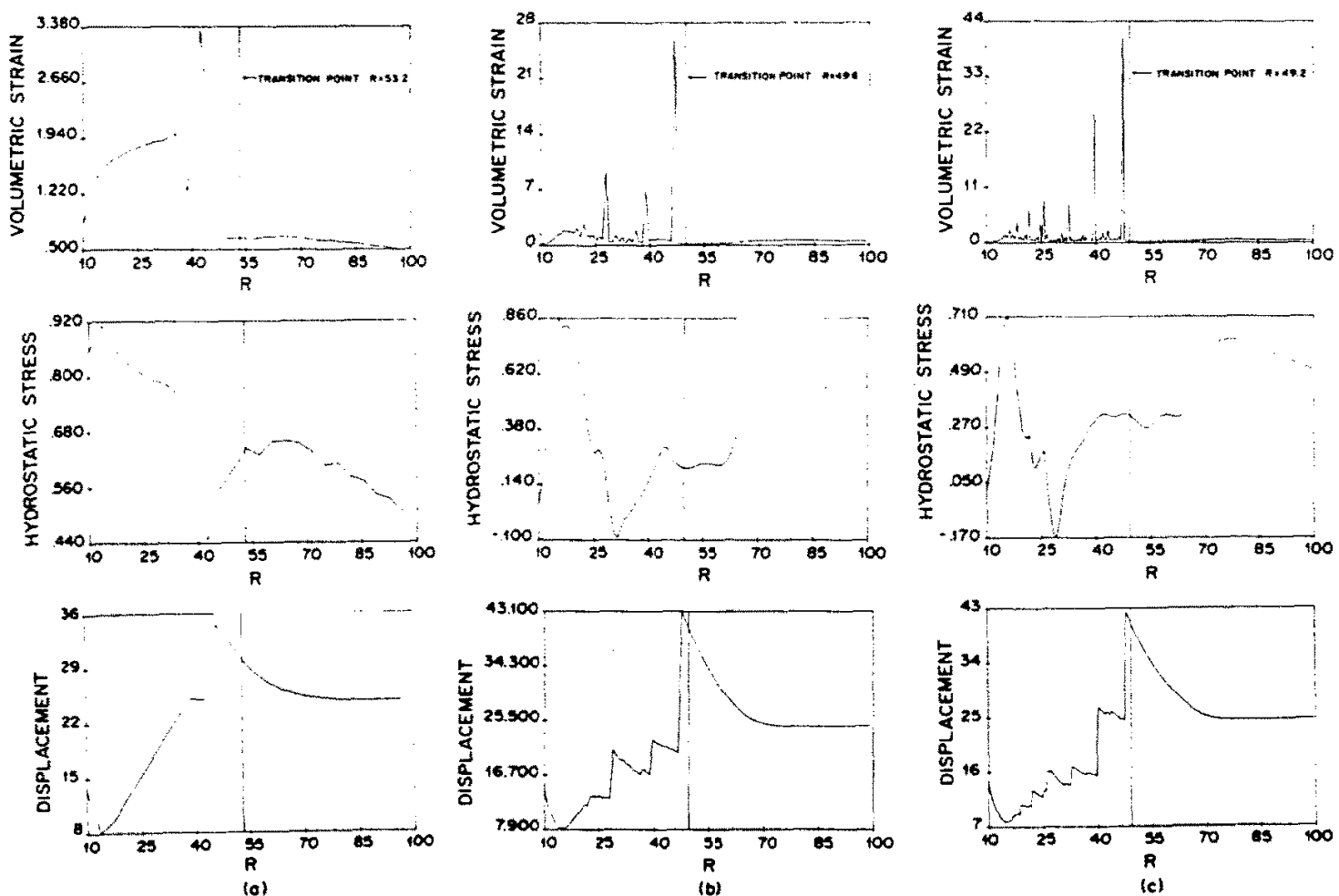

Fig. 13. Solutions to spherical wave problem for various meshes with damping without damping cutoff for meshes of (a) 25 elements. (b) 100 elements, and (c) 200 elements. 
ening for the purpose of achieving total behavior in which the strain softening terminates with a zero stress value[7]. The introduction of the local stresses through the coefficient $\gamma$. see eqn (3.7) is required for stability. For one-dimensional planar waves. it was shown[7] that stability is assurred for $y>0$. However, when $\gamma$ is small the noise is excessive. For one-dimensional planar waves, the value $y=0.1$ gave noise-free response. Curiously. the value of $\gamma$ for the cylindrical wave had to be higher $(\gamma=0.25)$ than the value for the spherical wave $(y=0.1)$ in order to obtain a noise-free response.

The values of the applied surface pressure are chosen as $p_{0}=0.708$ for the spherical wave and $p_{0}$ $=0.8$ for the cylindrical wave. For these boundary conditions, the wave propagating from the outer surface remains elastic until the wavefront reaches
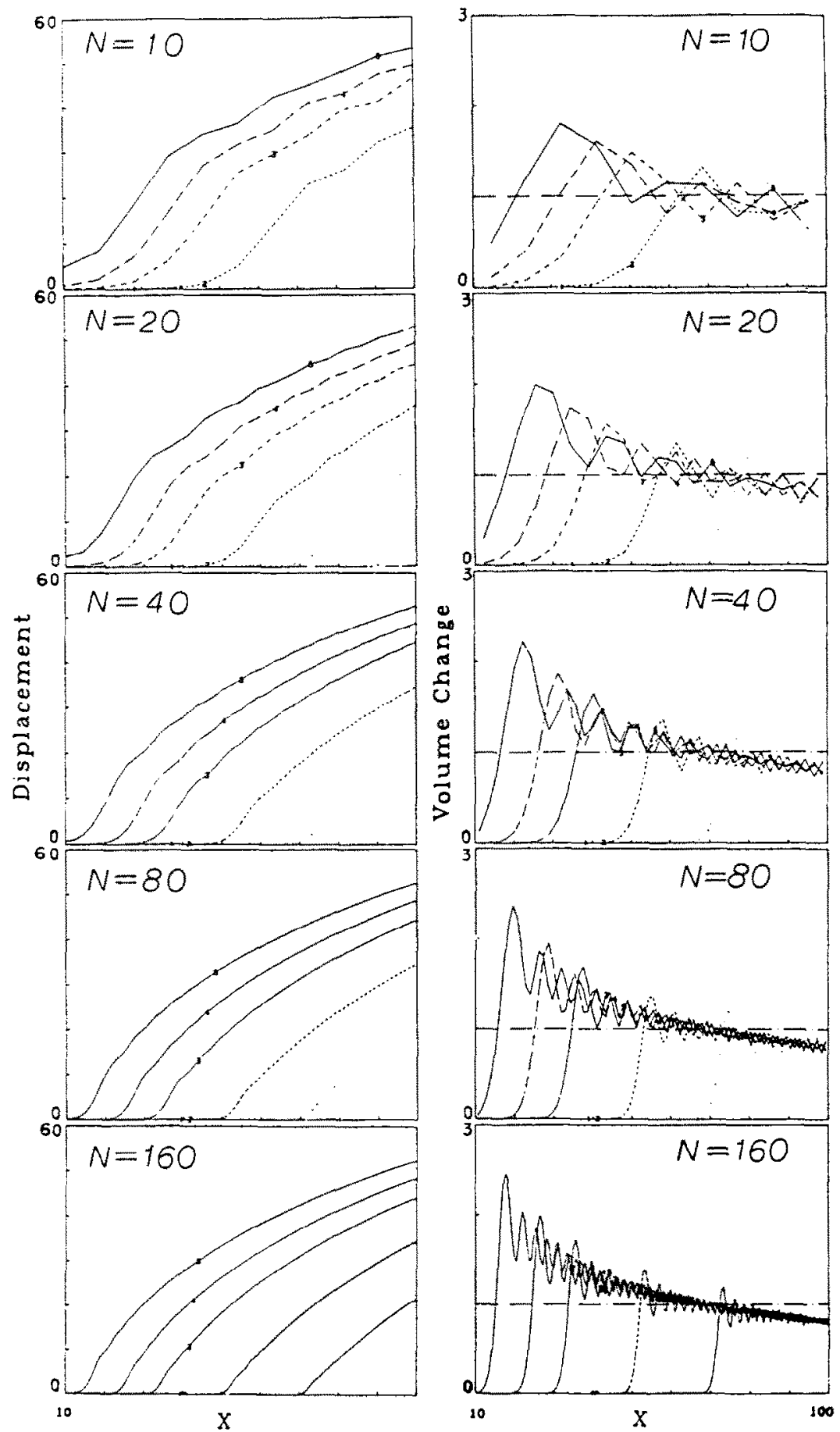

Fig. 14. Comparison of numerical solutions for elastic. linear spherical geometry with analytic solutions. 
$30 \%$ of the thickness $b-a$. The dimensions are $a$ $=10 . b=100 . L=b-a=90$.

In order to ascertain the mesh refinement necessary for this class of problems. elastic local solutions were obtained first. The convergence with

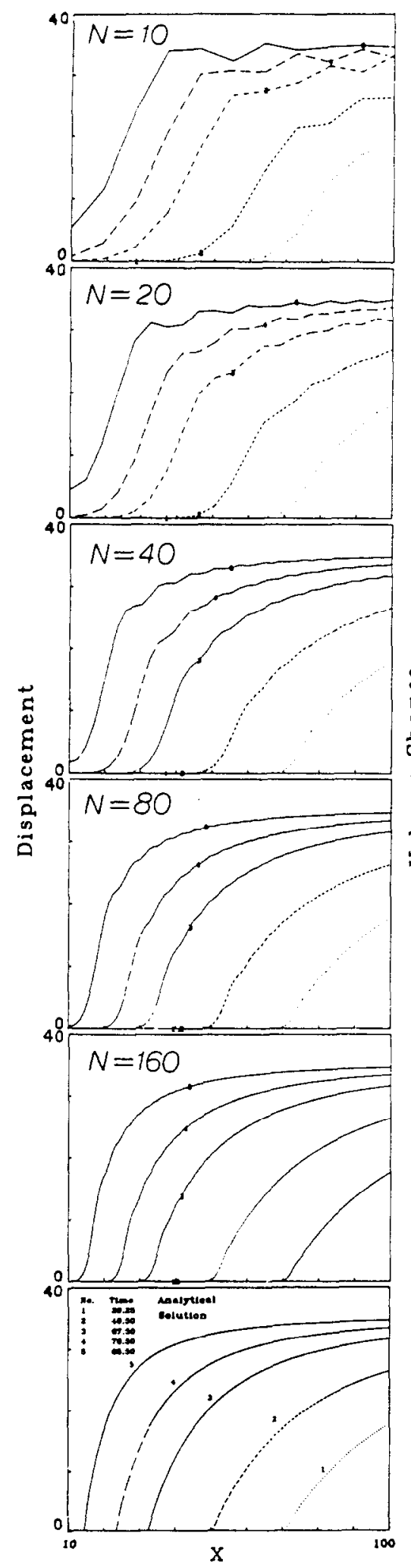

increasing numbers of elements $(N=10,20,40$. 80. 160) is shown in Figs. 14 and 15. The results converge to the exact solution given by eqn (5.9).

Subsequently, the problem was solved for a nonlocal continuum $\left(\epsilon_{p}=1 . \epsilon_{f}=5\right)$ with characteristic

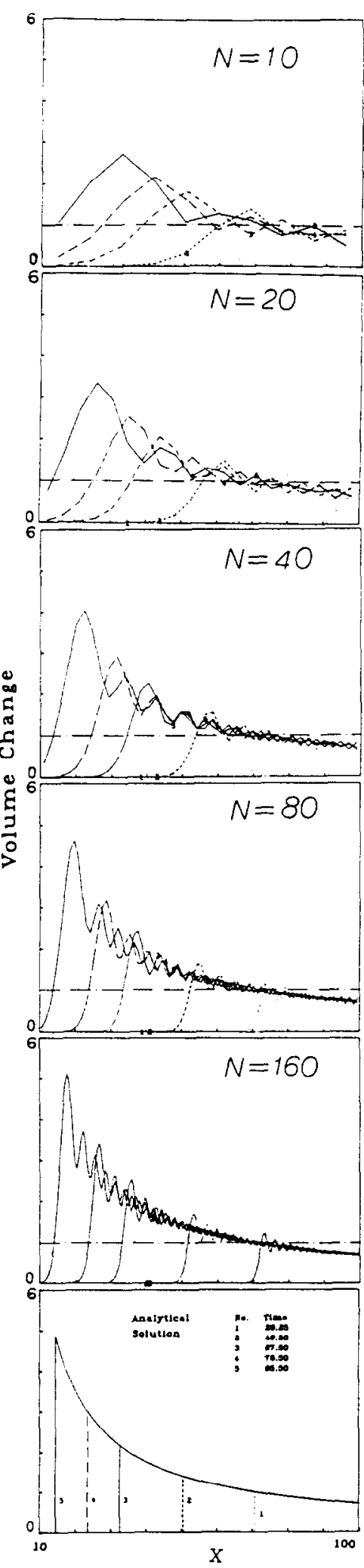

Fig. 15. Numerical solutions for elastic, linear cylindrical geometry. 
length $1=L / 10$. The results for progressively finer meshes $(N=10,20,40,80.160)$ are shown in Figs. 16-19. right columns. For comparison. the solution was also run for a local continuum $i l=h=L / N$ = variable with $N$ ) having the same stress-strain relation with strain softening: see Figs. 16-19, left columns. It is seen (Figs. $16-19)$ that the nonlocal solutions with strain softening converge well with increasing $N$. On the other hand, the corresponding local solutions do not converge at all.

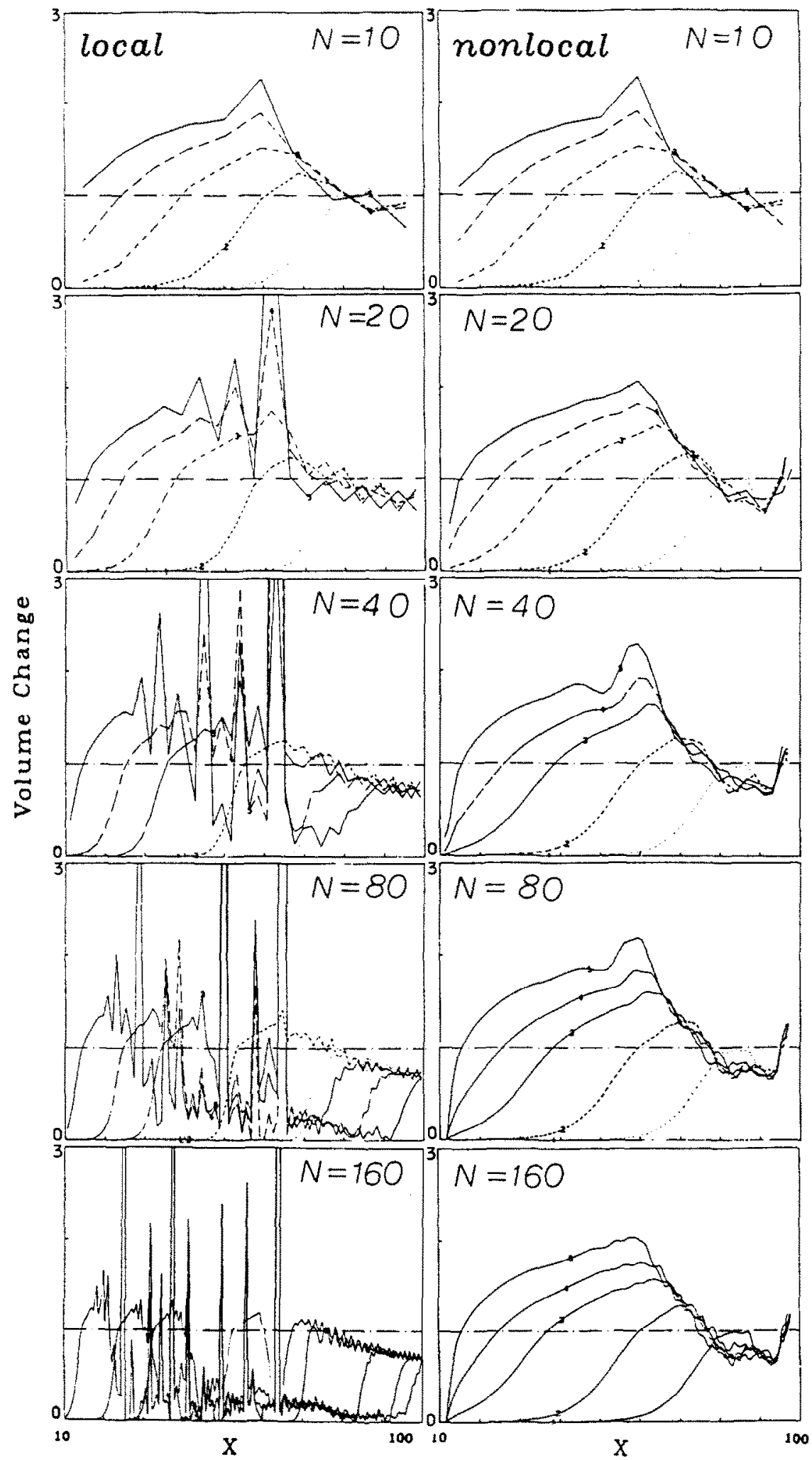

Fig. 16. Comparison of numerical results for local and nonlocal materials with spherical geometryvolume change. 


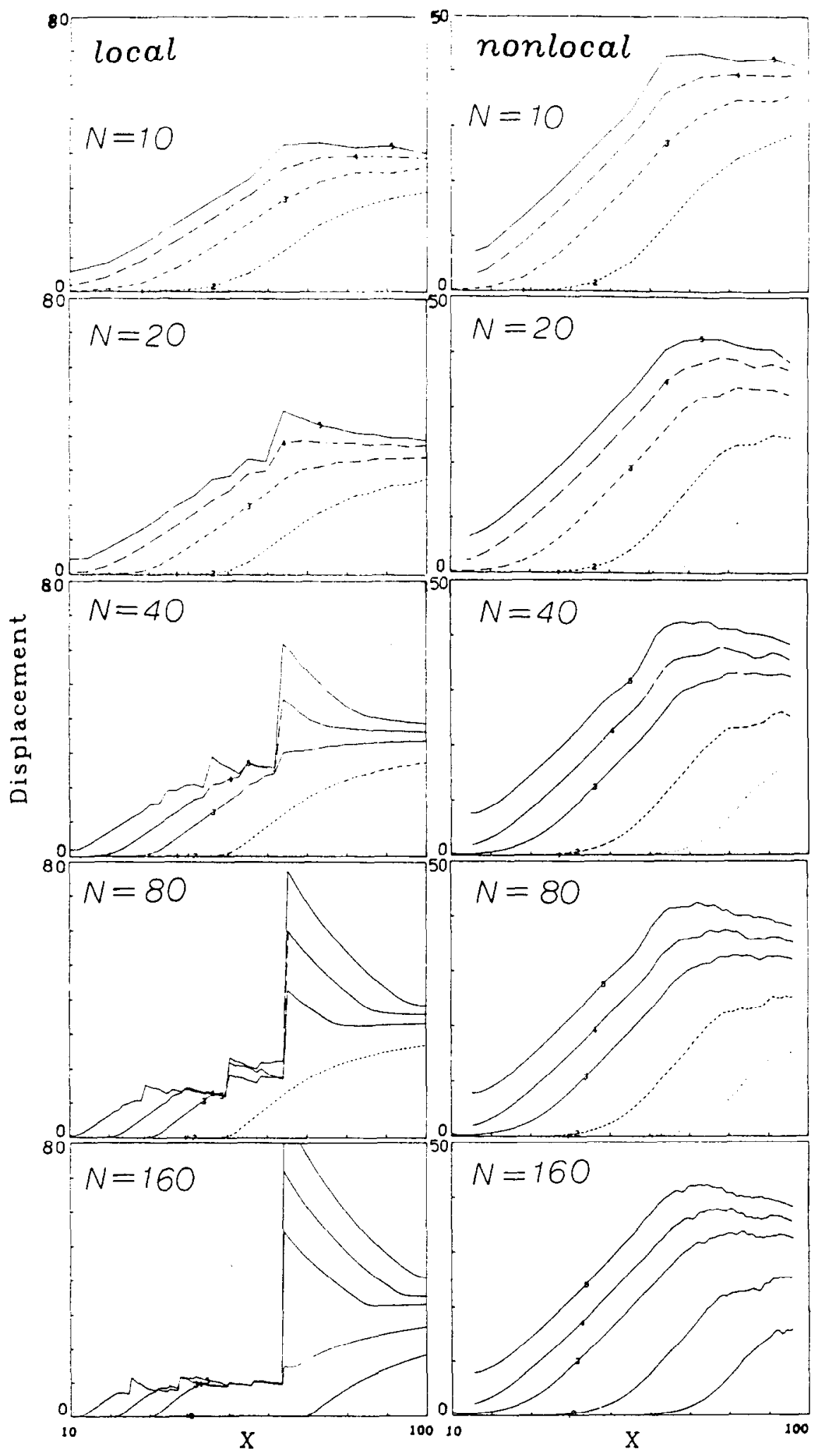

Fig. 17. Comparison of numerical results for local and nonlocal materials with spherical geometry radial displacement.

In Sec. 3, it was shown that for the uniaxial planar wave, finite-element solutions converge to the exact solution with a local strain-softening material. For spherical waves. no exact local material solution with strain softening has been found. As in the exact solution for the planar wave, the strainsoftening region appears to localize. producing singular strains, as can be seen from the spikes in Fig. 16 on the left. These singular strains begin to appear, as expected. when the strain at the wavefront 


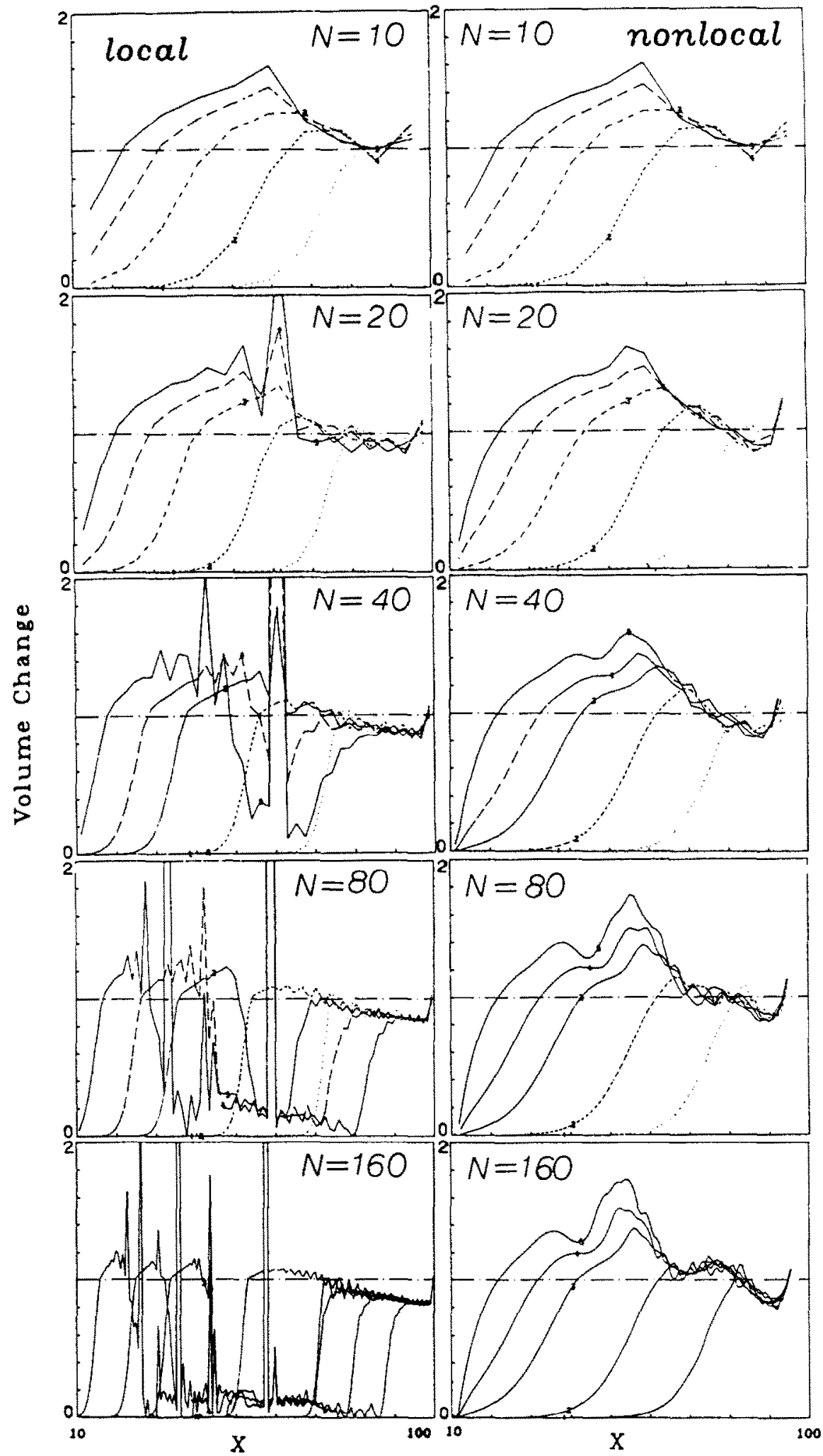

Fig. 18. Comparison of numerical results for local and nonlocal materials with cylindrical geometryvolume change.

reaches the strain at which strain softening begins. In contrast to the planar wave, there is not one point of localization but many, and they appear at different locations for different $N$ (i.e. for different mesh refinements), and the number of localization points increases with increasing mesh refinement. The impression is that of chaos. It is anticipated that if the loading is a ramp function in time. the set of localization points would be an infinitely dense set of discrete points. i.e. a Cantor set. The 


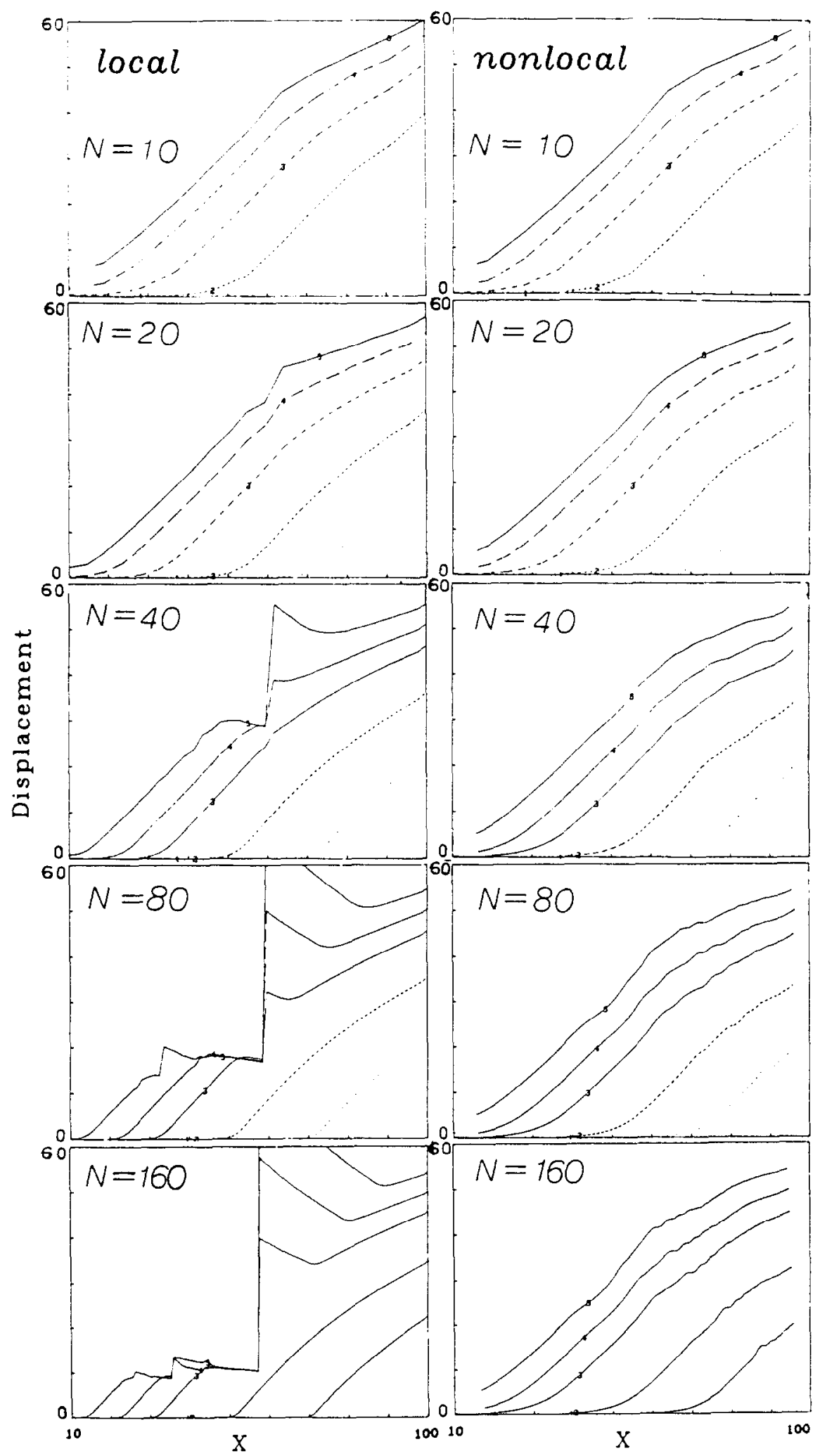

Fig. 19. Comparison of numerical results for local and nonlocal materials with cylindrical geometryradial displacement. 
reason for the appearance of multiple strain-softening points (the spikes in the left columns of Figs. 16 and 18 ) is that part of the strain step at the wavefront is transmitted across the surface of strain softening before the stress is reduced to zero. The part of the wave which has been transmitted then grows as the wavefront converges toward the center until the elastic limit $\epsilon_{p}$ is reached again. so the situation repeats itself.

\section{CONCLUSIONS}

The following are the major conclusions of the work:

1. Analytic solutions can be established for certain simple problems which include strain-softening materials. The solutions exhibit singular strain distributions but the rate of convergence of finite-element solutions is quite rapid.

2. In the spherical wave problem, numerical solutions of strain-softening models exhibit severe dependence on element mesh size. This is particularly true of field variables inside the surface of initial strain softening. Nonlocal models provide rapidly converging solutions to this problem.

3. A major difficulty of local laws with strain softening is that the energy dissipation vanishes. Thus, the failure process is not accompanied by energy dissipation. which is physically unrealistic.

4. Nonlocal laws provide a means for obtaining rapid convergence and finite energy dissipation in failure. However, the technology for efficiently implementing these techniques in large-scale, multidimensional problems remains to be developed.

Acknowledgments-We gratefully acknowledge the support of Air Force AFOSR Grant 83-0009 to Northwestern University.

\section{REFERENCES}

1. Z. P. Bażant and L. Cedolin, Fracture mechanics of reinforced concrete. J. Engng Mech. Div. ASCE 106, 1287-1306 (1980); Discussion and Closure in 108, 464471 (1982).

2. Z. P. Bažant, Instability, ductility and size effect in strain-softening concrete. J. Engng Mech. Div. ASCE 102, EM2, 331-334 (1976); discussion 103, 357-358, 775-777 (1977); 104, 501-502 (1978).

3. S. H. Marchertas, S. H. Fistedis, Z. P. Bažant, and T. Belytschko. Analysis and application of prestressed concrete reactor vessels for LMFBR containment. Nucl. Engng Des. 49, 155-174 (1978).

4. I. Sandler and J. Wright, Summary of strain-softening. In Theoretical Foundations for Large-Scale Computations of Nonlinear Material Behavior, DARPA-NSF Workshop (Edited by S. Nemat-Nasser). Northwestern University (1984).

5. T. Belytschko, Discussion of I. Sandler in Theoretical Foundations for Large-Scale Computations of Nonlinear Material Behavior, DARPA-NSF Workshop (Edited by S. Nemat-Nasser), pp. 285-315. Northwestern University (1984).

6. Z. P. Bazant and T. B. Belytschko, Wave Propagation in Strain-Softening Bar: Exact Solution. Report No. 83-10/401w to DNA. Center for Concrete and Geomaterials, Northwestern University, Evanston, Illinois, Oct. 1983. Also, J. Engng .Mech. Div. ASCE, 111, 381-389 (1985)

7. Z. P. Bażant. T. B. Belytschko, and T.-P. Chang. Continuum theory for strain softening. J. Engng Mech. ASCE 110, 1666-1692 (1984).

8. Z. P. Bažant. Imbricate continuum and its variational derivation. J. Engng Wech. ASCE 110, 1593-1712 (1984)

9. E. Kröner, Elasticity theory of materials with long range cohesive forces. Int. J. Solids Struct. 3, 731742 (1967).

10. J. A. Krumhansl, Some Considerations of the Relation Between Solid State Physics and Generalized Continuum Mechanics. Mechanics of Generalized Continua (Edited by E. Kröner), pp. 198-311. Springer-Verlag, Berlin (1968)

11. I. A. Kunin, The Theory of Elastic Media with Microstructure and the Theory of Dislocations. Mechanics of Generalized Continua (Edited by $\mathrm{E}$. Kröner), pp. 321-328. Springer-Verlag, Berlin (1968).

12. A. C. Eringen, Nonlocal polar elastic continua. Int. J. Engng Sci. 10, 1-16 (1972).

13. A. C. Eringen and D. G. B. Edelen, On nonlocal elasticity. Int. J. Engng Sci. 10, 233-248 (1972).

14. A. C. Eringen and N. Ari, Nonlocal stress field at Griffith crack. Cryst. Latt. Def. Amorph. Mat. 10,3338 (1983).

15. D. C. B. Edelen, Non-local variational mechanics IStationarity conditions with one unknown. Int. $J$. Engng Sci. 7, 269-285 (1969).

16. Z. P. Bażant and T.-P. Chang, Nonlocal Continuum and Strain-Averaging Rules, Report No. 83-11/401i, Center for Concrete and Geomaterials. Northwestern University, Evanston, Illinois, Nov. 1983; also J. Engng Mech. ASCE (in press).

17. J. Hadamard, Leçons sur la propagation des ondes, Chapt. VI. Hermann et cie, Paris (1903).

18. G. I. Taylor, The testing of materials at high rates of loading. J. Inst. Civ. Engng 26, 486-519 (1946).

19. F. H. Wu and L. B. Freund, Deformation trapping due to thermoplastic instability in one-dimensional wave propagation. J. Mech. Phys. Solids 32(2), 119132 (1984).

20. J. D. Achenbach, Wave Propagation in Elastic Solids. North-Holland, Amsterdam (1973).

\section{APPENDIX A}

Numerical algorithm for spherical and cylindrical waves (1) Read $a, b, l, n . \Delta t$ (time step), $N_{c}, \bar{N}_{c}, N_{h}, N_{t}$ (number of local elements, imbricate elements, nodes, and time steps, respectively), $\gamma$. and $p_{i}$. Generate arrays $\hat{k}(i) . \dot{m}(i)$. $l_{e}(i), x_{e}(i), \bar{k}(i), \bar{m}(i), \bar{l}_{e}(i), \bar{x}_{c}(i)$ giving the number of the left and right nodes of the $i$ th local or imbricate element. its length, and its coordinate at the center of element. Also generate externally applied nodal forces $f_{h . t}$. Initialize as zero the values (for $r=1$ ) of $i^{\prime} k, \mu_{h}, \tau_{r h}, \tau_{k h}, \sigma_{1 k,}, \max \epsilon_{h}$. $\max \bar{\epsilon}_{k}, \tau_{\max } \ldots k, \sigma_{\max }$, for all $k=1 \ldots, N_{\text {. }}$ (local elements) and $k=1 \ldots \ldots \bar{N}_{c}$ (imbricate elements).

(2) DO $8, r=2, \ldots N_{1}$.

(3) Initialize nodal forces $F_{k}=0 . f_{l}=0$ (for all nodes. $\left.k=1, \ldots, N_{k}\right)$.

(4) DO $5 i=1, \ldots . N_{c}$ (local elements).

(5) Set $k=\dot{k}(i), m=\dot{m}(i), x=x_{r}(i)$ and evaluate $\Delta \epsilon_{\mathrm{r} i}=$ $\left(v_{m}-v^{\prime}\right) \Delta t / h, \epsilon_{x i} \leftarrow \epsilon_{v i}+\Delta \epsilon_{v i} \Delta \epsilon_{v i}=\left(v_{\prime \prime}+v^{\prime} h\right) \Delta t / 2 x$, $\epsilon_{y i} \leftarrow \epsilon_{v i}+\Delta \epsilon_{i v}$. For spherical wave $\Delta \epsilon_{i}=J \epsilon_{x i}+2 \Delta \epsilon_{v i}$, and for cylindrical wave $J \epsilon_{i} \leftarrow \Delta \epsilon_{i j}+\Delta \epsilon_{i j}: \epsilon_{i} \leftarrow \epsilon_{i}+$ $\Delta \epsilon_{i}$. Then call a subroutine which determines the incremental (tangential) moduli $K_{i}, G_{i}$ for the local elements from their strains $\epsilon_{x i}, \epsilon_{v i}, \epsilon_{i}$ and also decides whether virgin loading. unloading. or reloading applies. Then calculate $\Delta \tau_{x i}=K_{i} \Delta \epsilon_{i}+2 G_{i} e_{x i} . \Delta \tau_{v i}=K_{i} \Delta \epsilon_{i}+2 G_{i} e_{v i}, T_{x i} \leftarrow$ 
$T_{1,}+\Delta \tau_{1, \ldots} \tau_{1,}=\tau_{1,}-\Delta \tau_{1,}$. Then calculate all the local nodal forces at element nodes $h$ and $m$ (Fig. 10c).

$\Delta f_{k}=-\gamma\left[T_{x i} x^{2}-\tau_{y i}\left(x-\frac{1}{2} h\right) h\right]$.

(spherical) (Al)

$\Delta f_{m}=\gamma\left[i_{x i} x^{2}+\tau_{y i}(x+\lfloor h) h]\right.$.

$$
\Delta f_{h}=y\left(\bar{\tau}_{1} x-\frac{1}{2} /\left(\bar{i}_{1 i}\right)\right. \text {. }
$$

(cylindrical) (A2)

$$
\left.\Delta f_{\mu \prime}=\gamma\left(\tau_{11} x+\frac{1}{2} / \tau_{w i}\right)\right) \text {. }
$$

These forces are accumulated at each node, $f_{k} \leftarrow f_{k}+$ $\Delta f_{k} . f_{m} \leftarrow f_{m}+\Delta f_{m}$.

(6) DO 7. $i=1, \ldots, \bar{N}_{e}$ (imbricate elements).

(7) Set $k=\bar{k}(i), m=\bar{m}(i), l=\bar{l}_{e}(i), x=\bar{x}_{e}(i)$ and evaluate $\Delta \bar{\epsilon}_{x i} \leftarrow\left(v_{m}-v_{k}\right) \Delta t / l, \bar{\epsilon}_{x i} \leftarrow \bar{\epsilon}_{x i}+\Delta \bar{\epsilon}_{x i}, \Delta \bar{\epsilon}_{y i} \leftarrow\left(v_{m}+\right.$ $\left.v_{k}\right) \Delta t / 2 x, \bar{\epsilon}_{y i} \leftarrow \bar{\epsilon}_{y i}+\Delta \bar{\epsilon}_{v i}$. For spherical wave $\Delta \bar{\epsilon}_{i}=\Delta \bar{\epsilon}_{x i}$ $+2 \Delta \bar{\epsilon}_{y i}$ and for cylindrical wave $\overline{\boldsymbol{\epsilon}}_{i}=\Delta \overline{\boldsymbol{\epsilon}}_{x i}+\Delta \overline{\boldsymbol{\epsilon}}_{y i} ; \overline{\boldsymbol{\epsilon}}_{i}=$ $\bar{\epsilon}_{i}+\Delta \bar{\epsilon}_{i}$. Then call a subroutine which determines the incremental (tangential) moduli $\bar{K}_{i}, \bar{G}_{i}$ for the imbricate elements from their mean strains $\overline{\boldsymbol{\epsilon}}_{x i}, \overline{\boldsymbol{\epsilon}}_{y i}, \overline{\boldsymbol{\epsilon}}_{i}$ and also decides whether virgin loading, unloading, or reloading applies. Then calculate $\Delta \sigma_{x i}=K_{i} \Delta \epsilon_{i}+2 G_{i} \Delta e_{x i}, \Delta \sigma_{y i}=$ $\left.\left.\bar{K}_{i}\right\lrcorner \epsilon_{i}+2 G_{i}\right\lrcorner e_{v i}, \sigma_{x i} \leftarrow \sigma_{x i}+\Delta \sigma_{x i}, \sigma_{v i} \leftarrow \sigma_{v i}+\Delta \sigma_{v i}$.
Then calculate all the nonlocal nodal forces at element nodes $b$ and $m$ (Fig. 10d).

$\Delta F_{\alpha}=-\frac{1-\gamma}{n}\left[\sigma_{x i} x^{2}-\sigma_{y i}\left(x-\frac{h}{2}\right) h\right]$.

(spherical) (A3)

$\Delta F_{m}=\frac{1-\gamma}{2}\left[\sigma_{x i}{ }^{2}+\sigma_{v i}\left(x+\frac{h}{2}\right) h\right]$.

$\Delta F_{k}=-\frac{1-\gamma}{n}\left(\sigma_{x, 1} x-\frac{h}{2} \sigma_{y i}\right)$.

(cylindrical) (A4)

$\Delta F_{m}=\frac{1-\gamma}{n}\left(\sigma_{x i} x+\frac{h}{2} \sigma_{y i}\right)$.

These forces are then accumulated at each node: $F_{A} \leftarrow F_{A}$ $\left.+\Delta F_{h} . F_{m} \leftarrow F_{m}+\right\lrcorner F_{m}$.

(8) DO 8, $k=1, \ldots N_{k}$ (all nodes)

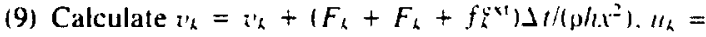
$u_{k}+\Delta t^{\prime \prime} k$.

For more detailed explanations. a similar algorithm for planar stress wave given in Ref. [7] may be consulted. It may be checked that the sum of the nodal forces given by eqns $(A \mid)-(A 4)$ on one node yields a second-order discrete approximation of the continuum equation of motion $(5,8)$. 